\title{
A TEXT MINING RESEARCH BASED ON LDA TOPIC MODELLING
}

\author{
Zhou Tong $^{1}$ and Haiyi Zhang ${ }^{2}$ \\ Jodrey School of Computer Science, Acadia University, Wolfville, NS, Canada \\ ${ }^{1}$ zhoutongeacadiau.ca \\ ${ }^{2}$ haiyi.zhangeacadiau.ca
}

\begin{abstract}
A Large number of digital text information is generated every day. Effectively searching, managing and exploring the text data has become a main task. In this paper, we first represent an introduction to text mining and a probabilistic topic model Latent Dirichlet allocation. Then two experiments are proposed - Wikipedia articles and users' tweets topic modelling. The former one builds up a document topic model, aiming to a topic perspective solution on searching, exploring and recommending articles. The latter one sets up a user topic model, providing a full research and analysis over Twitter users' interest. The experiment process including data collecting, data pre-processing and model training is fully documented and commented. Further more, the conclusion and application of this paper could be a useful computation tool for social and business research.
\end{abstract}

\section{KEYWORDS}

topic model, LDA, text mining, probabilistic model

\section{INTRODUCTION}

As computers and Internet are widely used in almost every area, more and more information is digitized and stored online in the form of news, blogs, and social networks. Since the amount of the information is exploded to astronomical figures, searching and exploring the data has become the main problem. Our research is intended to design a new computational tool based on topic models using text mining techniques to organize, search and analyse the vast amounts of data, providing a better way understanding and mining the information.

\section{BACKGROUND}

\subsection{Text Mining}

Text mining is the process of deriving high-quality information from text [1]. Text mining usually involves the process of structuring the input text, finding patterns within the structured data, and finally evaluation and interpretation of the output. Typical text mining tasks include text categorization, text clustering, document summarization, keyword extraction and etc. In this 
research, statistical and machine learning techniques will be used to mine meaningful information and explore data analysis.

\subsection{Topic Modelling}

In machine learning and natural language processing, topic models are generative models, which provide a probabilistic framework [2]. Topic modelling methods are generally used for automatically organizing, understanding, searching, and summarizing large electronic archives.

The "topics" signifies the hidden, to be estimated, variable relations that link words in a vocabulary and their occurrence in documents. A document is seen as a mixture of topics. Topic models discover the hidden themes through out the collection and annotate the documents according to those themes. Each word is seen as drawn from one of those topics. Finally, A document coverage distribution of topics is generated and it provides a new way to explore the data on the perspective of topics.

\subsection{Latent Dirichlet Allocation}

Latent Dirichlet allocation (LDA) is a generative model that allows sets of observations to be explained by unobserved groups that explain why some parts of the data are similar [3]. LDA has made a big impact in the fields of natural language processing and statistical machine learning and has quickly become one of the most popular probabilistic text modelling techniques in machine learning.

Intuitively in LDA, documents exhibit multiple topics [4]. In text pre-processing, we exclude punctuation and stop words (such as, "if", "the", or "on", which contain little topical content). Therefore, each document is regarded as a mixture of corpus-wide topics. A topic is a distribution over a fixed vocabulary. These topics are generated from the collection of documents [5]. For example, the sports topic has word "football", "hockey" with high probability and the computer topic has word "data", "network" with high probability. Then, a collection of documents has probability distribution over topics, where each word is regarded as drawn from one of those topics. With this document probability distribution over each topic, we will know how much each topic is involved in a document, meaning which topics a document is mainly talking about.

A graphical model for LDA is shown in Figure 1:

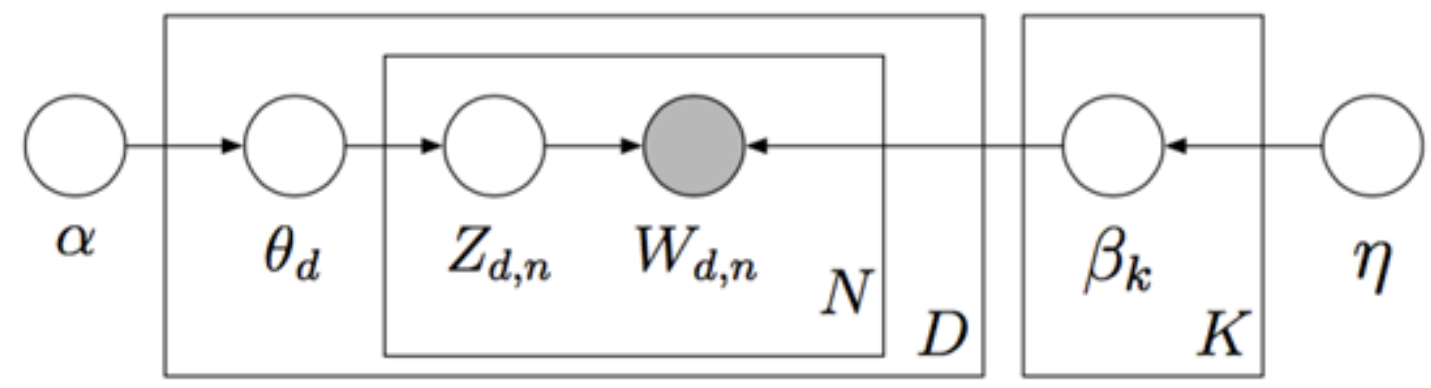

Figure 1. Graphic model for Latent Dirichlet allocation 
As the figure illustrated, we can describe LDA more formally with the following notation. First, $\alpha$ and $\eta$ are proportion parameter and topic parameter, respectively. The topics are $\beta_{1: K}$, where each $\beta_{k}$ is a distribution over the vocabulary. The topic proportion for the $d$ th document are $\theta_{d}$ , where $\theta_{d, k}$ is the topic proportion for topic $k$ in document $d$. The topic assignments for the $d$ th document are $Z_{d}$, where $Z_{d, n}$ is the topic assignment for the $n$th word in document $d$. Finally, the observed words for document $d$ are $w_{d}$, where $w_{d, n}$ is the $n$th word in document $d$, which is an element from the fixed vocabulary.

With this notation, the generative process for LDA corresponds to the following joint distribution of the hidden and observed variables:

$$
p\left(\beta_{1: K}, \theta_{1: D}, z_{1: D}, w_{1: D}\right)=\prod_{i=1}^{K} p\left(\beta_{i}\right) \prod_{d=1}^{D} p\left(\theta_{d}\right)\left(\prod_{n=1}^{N} p\left(z_{d, n} \mid \theta_{d}\right) p\left(w_{d, n} \mid \beta_{1: K}, z_{d, n}\right)\right)
$$

Notice that this distribution specifies a number of dependencies. The topic assignment $Z_{d, n}$ depends on the per-document topic distribution $\theta_{d}$; and the word $w_{d, n}$ depends on all of the topics $\beta_{1: K}$ and the topic assignment $Z_{d, n}$.

\subsection{Jensen-Shannon Divergence}

In probability theory and statistics, the Jensen-Shannon divergence is a popular method of measuring the similarity between two probability distributions. It is also known as information radius or total divergence to the average. It is based on the Kullback-Leibler divergence. The square root of the Jensen-Shannon divergence is a metric often referred to as Jensen-Shannon distance [6].

For discrete probability distributions $P$ and $Q$, Kullback-Leibler divergence of $Q$ from $P$ is defined to be:

$$
D_{K L}(P \| Q)=\sum_{i} P(i) \log \frac{P(i)}{Q(i)}
$$

So, the Jensen-Shannon divergence of $Q$ from $P$ is defined by:

$$
J S D(P \| Q)=\frac{1}{2} D_{K L}(P \| M)+\frac{1}{2} D_{K L}(Q \| M)
$$

where $M=\frac{1}{2}(P+Q)$

Jensen-Shannon divergence measures the similarity between two distributions. By applying Jensen-Shannon divergence to the topic assignment for the $d$ th document $Z_{d}$, it will allow us to measure the distance and similarity between each document. 


\section{DESIGNS AND EXPERIMENTS}

In order to apply topic modelling and propose a new text mining solution on topics, we have designed two experiments fulfilling our goal. The first one is to use topic modelling manage and explore Wikipedia, and the second is a Twitter application on topic modelling. The former is a new solution on a typical problem and the latter is building up a new model on Twitter data analysis.

\subsection{Wikipedia on Topic Modelling}

\subsubsection{Experiment Overview}

Wikipedia is a free-access, free-content Internet encyclopaedia, supported by non-profit Wikimedia Foundation. It has millions of articles for people to search, explore or even edit. In this experiment, the text data is from simplified Wikipedia (English version) with over 200,000 articles. By applying Latent Dirichlet allocation (LDA) and topic modelling, a solution of topic searching, exploring and recommending system will be achieved.

\subsubsection{Data Pre-processing}

The simplified Wikipedia English version is free for download from Wikipedia Foundation database backup dumps. The backup is in a format of XML. The first step of data pre-processing is to parse the XML file and extract the text data. $\mathrm{R}$ package $X M L$ provides a series of function parsing XML file. By using those functions, we will get a relatively clear data of all the articles in a data frame.

The next step is text-cleaning process. The purpose of text cleaning is to simplify the text data, eliminating as much as possible language dependent factors. Articles are written in natural language for human to understand. But in text mining, those data are not always easy for computers to process. In this experiment, there are three steps in text cleaning:

- Tokenization: a document is treated as a string, removing all the punctuations and then partitioned into a list of tokens.

- $\quad$ Removing stop words: stop words such as "the", "if", "and" ... are frequently occurring but no significant meanings which need to be removed.

- Stemming word: stemming word that converts different word form into similar canonical form. For example, computing to compute, happiness to happy. This process reduces the data redundancy and simplifies the later computation [7].

\subsubsection{Model Training}

The training process requires $\mathrm{R}$ package topicmodels with its package dependencies ( $\mathrm{tm}$ and others) to be loaded. An LDA model of simplified English Wikipedia on a sample of 1000 articles with more than 1000 characters, returned after 2000 iterations of Gibbs sampling, with $K=50$ topics, and Dirichlet hyper-parameters $\beta=0.1$ and $\alpha=50 / K$. Meanwhile, topic distribution coverage for each document is generated. This distribution represents how much each 
document is related to each topic. A new way of search and explore documents over topics can be implemented.

Table 1. A few selected topics generated from Wikipedia topic distribution

\begin{tabular}{|c|c|c|c|c|}
\hline Topic 2 & Topic 13 & Topic 22 & Topic 31 & Topic 46 \\
\hline athlete & album & universal & movie & hurricane \\
\hline olympia & song & college & categories & major \\
\hline field & music & school & fiction & season \\
\hline summer & record & categories & solstice & minor \\
\hline track & band & new & alien & key \\
\hline men & release & economic & star & storm \\
\hline metre & single & institut & sun & tropic \\
\hline image & rock & educate & direct & chord \\
\hline women & singer & science & drama & verse \\
\hline medal & pop & work & southern & end \\
\hline
\end{tabular}

Table 1 shows five selected topic terms after the model is trained, where top ten terms are listed for each topic. With LDA training, the terms in the same topic tend to be similar. Formally speaking, they are highly associated. For example, topic 13 is about music, topic 22 is about education and topic 46 is about weather. This topic distribution provides a way to search topic and explore between topics in order to find the document the user is looking for.

After the model is built, Jensen-Shannon divergence is applied to calculate the similarity of each distribution. Sorting the similarity of one document between every other distribution, a topic recommender system can be implemented.

\subsubsection{Results}

Here is an example of article Light from the experiment. The original article is shown in the left part of Figure 2, which can be also accessed in simple Wikipedia online (the data for this experiment is retrieved as a backup version on 11/1/2016). After the model is trained, we got a series of article distribution over each topic. The right part of Figure 2 is the bar plot of article light topics distribution. In total of 50 topics, we can easily find there are 3 topics with obviously high probabilities - topic 47, topic 45 and topic 16. Table 2 shows the top 5 probabilities topics. These probabilities are how tightly this article is associated with each topic. Table 3 shows the terms in these 5 topics with 10 terms each.
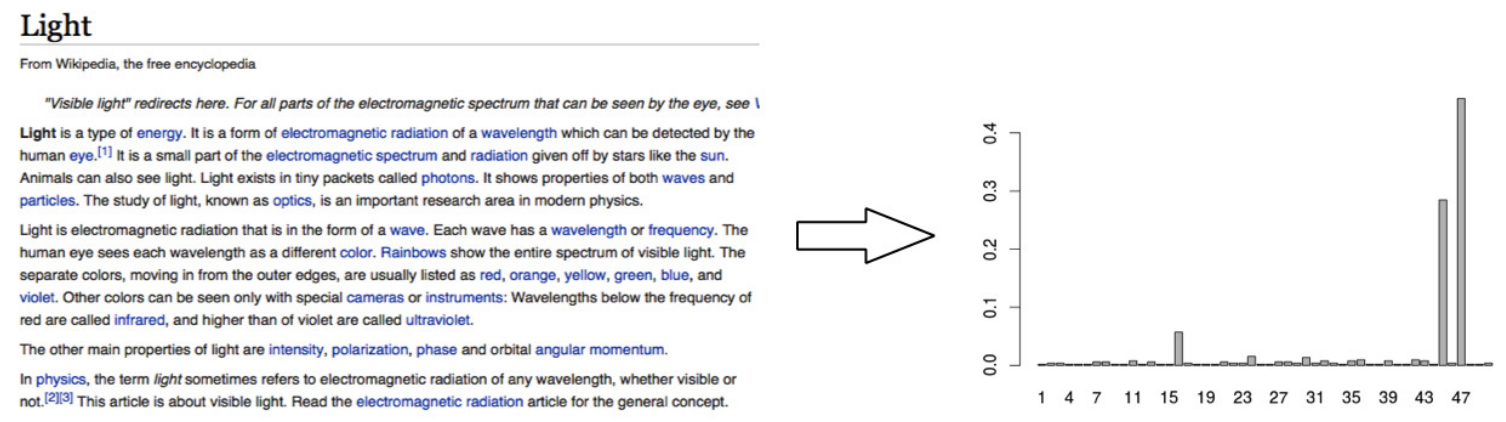

Figure 2. Topics Distribution of Article Light 
Table 2. Top 5 Topic Probabilities of Article Light

\begin{tabular}{|c|c|c|c|c|c|}
\hline Topics & Topic 47 & Topic 45 & Topic 16 & Topic 24 & Topic 30 \\
\hline Probabilities & 0.05692600 & 0.45920304 & 0.28462998 & 0.01518027 & 0.01328273 \\
\hline
\end{tabular}

Table 3. Top 5 Topic Terms of Article Light

\begin{tabular}{|c|c|c|c|c|}
\hline Topic 47 & Topic 45 & Topic 16 & Topic 24 & Topic 30 \\
\hline light & use & color & computable & cleaner \\
\hline beamline & one & style & equation & people \\
\hline beam & also & background & fluid & use \\
\hline radiated & can & hex & categories & clean \\
\hline don & people & rgb & image & make \\
\hline synchrotron & mania & magenta & program & chemical \\
\hline wavelength & call & ffffff & protocol & thing \\
\hline physical & time & fuchsia & mathematical & paint \\
\hline station & two & red & function & made \\
\hline carlo & like & pink & design & put \\
\hline
\end{tabular}

So just like this example, every article of the whole collection is represented as a vector of probabilities over 50 topics. This is the core data of our model, where we can do all sorts of applications. Here is an example of finding the most related article of article Light. We will use Jensen-Shannon divergence to calculate the distance between article Light and every other article. The shortest distance will be the most related article. After calculating the distance, Table 4 shows the top 10 of the shortest articles. To be noted, the distance should have been the square root of the distance below, but to simplify the calculation and higher the accuracy, we will stay with the squared number, as there would be no difference on comparing the closet distance.

Table 4. Top 10 Shortest Distance of Article Light

\begin{tabular}{|c|c|c|c|c|c|}
\hline Articles & Article 855 & Article 820 & Article 837 & Article 299 & Article 911 \\
\hline Distance $^{2}$ & 0.0000000 & 0.2271741 & 0.3404084 & 0.3881467 & 0.4583745 \\
\hline Articles & Article 341 & Article 287 & Article 328 & Article 544 & Article 606 \\
\hline Distance $^{2}$ & 0.4671845 & 0.4728383 & 0.4802241 & 0.4803357 & 0.4874499 \\
\hline
\end{tabular}

As shown on Table 4, these 10 articles are the closest distance with Article Light. The distance of Article 855 is 0, because it is article Light itself. So Article 820 is the closest distance with Light, meaning their contents most related. Meanwhile, what we get is a sorted list of closest distance and will also work if we require more than one most related article. This method is based on calculating the probabilities each meaningful word in the model. So the accuracy is much better than calculating the keywords, or titles, which is widely used on many documents management system. Lastly, if we look up the title of Article 820, it is Beamline. Based on the 2000 article dataset, it is a convincing answer to the most related article of Light.

\subsection{Twitter Data Analysis}

\subsubsection{Experiment Overview}

Twitter is an online social networking service that enables users to send and read short 140character messages called "tweets". Currently, Twitter has more than 332 million active users posting 340 million tweets a day. Twitter has a big impact in everyday life. Twitter mining is not 
only a big research task in computer science or statistics, but also a key factor on social and business research. In this experiment, by applying LDA and topic modelling, a deep research and analysis on Twitter users is proposed. A detail model of Twitter user's personality and preference will be inferred.

\subsubsection{Data Pre-processing}

Generally, a tweet from any twitter user is public and free to read for everyone. Having registered as a Twitter application developer will allow you to access all the tweets and a set of APIs to manipulate the data [8]. The first step is to collect tweets from the users. By using the APIs and $\mathrm{R}$ package twitteR, a sample of 10,000 valid users is gathered into a data frame. To improve the data quality, a standard is set up for the twitter user to be a "valid" user:

- The user profile is an unprotected, which means user's information and tweets is public to everyone. If the user sets the profile to be protected, his or her information cannot be gathered by developers.

- The user has at least 100 tweets. According to a statistical research from Twitter on January 2012, the average length of a tweet is 67.9 characters. Therefore, before pre-processing, there are 6790 characters for one user in a sample. Less than 100 tweets per user will lower the calculation quality.

- The user must use English as major language in the tweets. Some odd non-English word will not affect the model, but a number of total non-English users' data will longer the preprocessing time, add confusion to the topics, or even mess up the result.

The text cleaning process is basically the same as the Wikipedia experiment. Tokenization, stop words removing and word stemming is required in this process. However most tweets are oral and informal language, a few details need to be noticed:

- $\quad$ Some tweets may contain URLs, using hash tags on a topic, using @ to mention other users. In text cleaning process, these situations need particular functions to remove or parse.

- $\quad$ Some Internet terms, such as "LOL", "OMG", "BTW", are abbreviation of a phrase. Those terms can treat as stop words to delete.

- Some words are written as shorthand, such as "ppl" (people), "thx" (thanks), "fab" (fabulous). Those words need to stem to the original form.

\subsubsection{Model Training}

The training process is also similar to the Wikipedia experiment. However, Twitter data are formed with natural daily language, which has a narrow topic range compared to Wikipedia. The topics number is less and not with equally clear boundaries. Nevertheless, the topic model still has a good performance and the coverage distribution can easily illustrate the user's personal interest. Here is an experiment a sample of 100 twitter user with more than 100 tweets, returned after 2000 iterations of Gibbs sampling, with 30 topics. 
Table 5. A few selected topics generated from Twitter topic distribution

\begin{tabular}{|c|c|c|c|c|}
\hline Topic 1 & Topic 5 & Topic 9 & Topic 17 & Topic 28 \\
\hline halifax & check & stream & follow & weight \\
\hline nova & reward & live & win & loss \\
\hline scotia & one & league & enter & diet \\
\hline man & kangaroo & communities & canada & news \\
\hline refuge & new & check & retweet & lose \\
\hline media & facebook & ea & card & natural \\
\hline say & get & design & sunday & tip \\
\hline woman & post & weekend & away & plan \\
\hline central & photo & chat & chance & health \\
\hline student & coffee & gold & donate & techno \\
\hline
\end{tabular}

Table 2 shows five selected topics terms after the Twitter topic model is trained. Similar to the previous experiment, the LDA model has a good performance on dividing topics. But as we expected, twitter data is limited by the daily language that leads to less clear boundaries as Wikipedia topics. For example, in topic 5, it is hard to label this topic into a particular category. However, the Twitter application is successful on building up topic models over users, and it will certainly benefit the statistic analysis and even a big impact on social and business research.

\subsubsection{Results}

This experiment has a similar structure with the Wikipedia articles. Instead of each article, we will treat every user's tweets as an article. With the topic model, we can also calculate the distribution over each topic. Here is an example of Tim Cook's Twitter. As shown in Figure 3, the left part is the Twitter user, and the right part is the bar plot of topic distribution. This distribution represents what kind of topic the user talks more and more interested. Table 6 shows the top 5 topics of Tim Cook talks most about. By applying Jenson-Shannon divergence to calculate the distance, we can find the people that talks the most similar topics or even with similar personality.
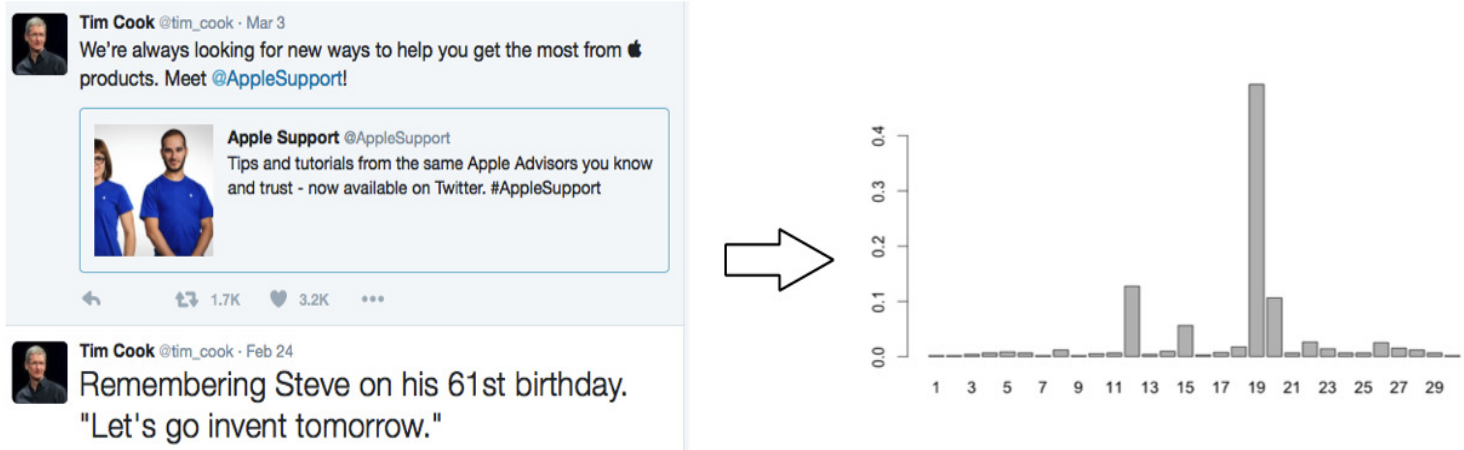

Figure 3. Topics Distribution of Twitter of Tim Cook 
Table 6. Top 5 Topic Terms of Tim Cook's Tweets

\begin{tabular}{|c|c|c|c|c|}
\hline Topic 19 & Topic 12 & Topic 20 & Topic 15 & Topic 22 \\
\hline day & new & apple & custom & summer \\
\hline today & great & iphone & service & student \\
\hline thank & canada & trump & expect & job \\
\hline will & join & deal & job & american \\
\hline great & congrat & say & product & wed \\
\hline time & congratulations & app & donet & still \\
\hline get & communities & product & photo & campus \\
\hline ea & forward & vs & price & fair \\
\hline can & proud & court & easier & act \\
\hline new & event & camera & facebook & hall \\
\hline
\end{tabular}

\section{CONCLUSIONS AND FUtURE WORK}

In this paper, an introduction of text mining and topic model LDA is represented. We proposed two experiments, which built up topic models on Wikipedia articles and Twitter users' tweets. A brief introduction of each experiment including overview, pre-processing and model training is given and analysed.

With these data and model foundation, a number of future works can be done for further research and experiment.

- As the limitation of the computation power, this research is based on a relatively small sample by the time we start writing. However, the result is quite convincing even with the small size. Applying to a larger dataset will more likely achieve better results.

- An application on topic modelling to manage, search and explore offline Wikipedia articles could be implemented.

- A full research on Twitter users' interest could be applied. Further more, this application could be a useful tool for social and business research

- In Twitter application on topic modelling, we ignore the pictures users posted. What if we can combine image processing and topic model to provide a better performance?

\section{REFERENCES}

[1] Martin Ponweiser (2012) Latent Dirichlet Allocation in R, Vienna University of Business and Economics.

[2] Bettina Grun, kurt Hornik (2011) "topicmodels: An R Package for Fitting Topic Model”, Journal of Statistical Software Vol. 40, No. 13.

[3] Qi Jing (2015) Searching for Economic Effects of User Specified Event Based on Topic Modelling and Event Reference, Jordery School of Computer Science, Acadia University.

[4] David M.Blei (2012) "Probabilistic Topic Models", Communications of the ACM Vol. 55, No. 4, pp77-84. 
[5] David M.Blei, John D. Lafferty (2006) "A Correlated Topic Model of Science”, Annals of Applied Statistics Vol. 1, No. 1, pp17-35.

[6] Jianhua Lin (1991) "Divergence Measures Based on the Shannon Entropy", IEEE Transactions on Information Theory Vol. 37, No. 1, pp145-151.

[7] Aurangzeb Khan, Baharum Baharudin, Lam Hong Lee, Khairullah khan (2010) "A Review of Machine Learning Algorithms for Text-Document Classification", Journal of Advances in Information Technology Vol. 1, No. 1, pp4-20.

[8] Yanchang Zhao (2015) R and Data Mining, http://www.rdatamining.com.

\section{AUTHORS}

Zhou Tong is currently a master student of computer science at Acadia University, Canada. His research is focusing on text mining.

Haiyi Zhang received his MS degree in 1990 from the Computer Science department of New Jersey Institute of Technology of USA, and his Ph.D in 1996 from Harbin Institute of Technology in China. He was a post-doctor in information department of ABO, Finland in 2000. His research interests are machine learning, data mining. He has more than 50 academic papers published. Currently he is an associate professor at Acadia University, Canada.

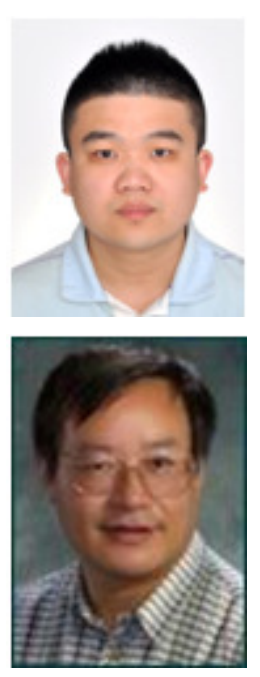

